\title{
Tricuspid valve repair and pulmonary valve replacement in adults with repaired tetralogy of Fallot
}

François Roubertie, MD, PhD, ${ }^{\mathrm{a}}$ Pierre-Emmanuel Séguéla, $\mathrm{MD},{ }^{\mathrm{b}}$ Zakaria Jalal, $\mathrm{MD},{ }^{\mathrm{b}}$ Xavier Iriart, $\mathrm{MD},{ }^{\mathrm{b}}$

Xavier Roques, MD, PhD, ${ }^{a}$ Bernard Kreitmann, MD, PhD, ${ }^{a}$ Mohammed Al-Yamani, MD, ${ }^{a}$

Xavier Pillois, $\mathrm{PhD},{ }^{b}$ and Jean-Benoît Thambo, $\mathrm{MD}, \mathrm{PhD}^{\mathrm{b}}$

\section{ABSTRACT}

Background: Pulmonary valve replacement (PVR) often is performed in adults with repaired tetralogy of Fallot (TOF). For patients who have tricuspid regurgitation (TR), tricuspid valve (TV) repair associated to PVR is still debated.

Objective: We sought to evaluate perioperative factors related to TV repair when performed at the time of PVR in patients with repaired TOF.

Methods: We retrospectively reviewed 104 patients with repaired TOF (or its equivalent) who underwent PVR (2002-2014).

Results: Mean age at initial complete correction and at PVR was $20.1 \pm 17.2$ months and $26.3 \pm 9.5$ years, respectively. Forty-one patients had significant preoperative TR: 24 were moderate (group M) and 17 were severe (group S). A total of 16 TV repair were performed ( 8 for each group). Moderate and severe tricuspid regurgitation observed in the first year following the initial complete repair were significantly associated with severe TR at PVR $(P<.001)$. In group $M$ patients, TR was improved regardless of TV repair, whereas, in group $S$, residual significant TR was reported in 7 patients who did not have TV repair. No cases were observed for patients who underwent concomitant TV repair $(P=.002)$. Among these patients with residual significant TR, 2 needed a tricuspid valve replacement. The functional status (New York Heart Association classification) of group $\mathrm{S}$ patients was significantly improved by concomitant TV repair.

Conclusions: In adults with repaired TOF, TV repair is a safe procedure when performed at the time of PVR. If, at mid-term follow-up, there is probably no benefit of TV repair when preoperative TR is moderate, TV repair may improve both tricuspid valve function and functional status of the patients in case of severe preoperative TR. (J Thorac Cardiovasc Surg 2017;154:214-23)

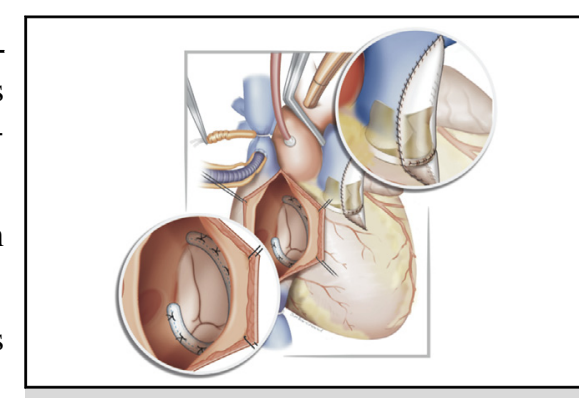

Tricuspid valve repair and pulmonary valve replacement in repaired tetralogy of Fallot.

\section{Central Message}

Tricuspid valve repair performed at the time of pulmonary valve replacement may be beneficial in selected adult patients with repaired tetralogy of Fallot.

\section{Perspective}

For adults with repaired tetralogy of Fallot and who will undergo pulmonary valve replacement, a tricuspid valve repair should strongly be considered in those with severe tricuspid valve regurgitation.

See Editorial Commentary page 224.

See Editorial page 212.
Usually, a tetralogy of Fallot (TOF) is repaired completely during infancy with a transannular patch that relieves right ventricle (RV) obstruction. Then, the right ventricular outflow tract (RVOT) usually grows proportionally with the child. Unvalved RVOT, however, induces long-term pulmonary regurgitation (PR) and causes chronic volume

\footnotetext{
From the Departments of ${ }^{\mathrm{a}}$ Cardiovascular Surgery and ${ }^{\mathrm{b}}$ Pediatric and Congenital Cardiology, Bordeaux University Hospital, Bordeaux, France.

Received for publication Jan 25, 2016; revisions received Nov 22, 2016; accepted for publication Dec 7, 2016; available ahead of print March 11, 2017.

Address for reprints: Pierre-Emmanuel Séguéla, MD, Department of Pediatric Cardiology, Hôpital Haut Lévêque-Bordeaux University Hospital, Ave de Magellan, 33600 Pessac, France (E-mail: peseguela@yahoo.fr). $0022-5223 / \$ 36.00$

Copyright (c) 2017 by The American Association for Thoracic Surgery http://dx.doi.org/10.1016/j.jtcvs.2016.12.062
}

overload of the RV. Resultant delayed RV dysfunction, propensities towards arrhythmia, and potential heart failure may necessitate, in adulthood, a pulmonary valve replacement (PVR). ${ }^{1}$ Although PVR in repaired TOF represents in adults a frequent surgical procedure of the RVOT, surgical management of an associated tricuspid regurgitation (TR), which is observed in up to $30 \%$ of patients, remains debated. ${ }^{2-4}$ Indeed, only scarce data on the surgical

Scanning this QR code will take you to a supplemental video for the article. 

Abbreviations and Acronyms
$\mathrm{CMR}=$ cardiac magnetic resonance imaging
NYHA $=$ New York Heart Association
$\mathrm{PR}=$ pulmonary regurgitation
PVR = pulmonary valve replacement
$\mathrm{RV}=$ right ventricle
RVOT $=$ right ventricular outflow tract
TOF $=$ tetralogy of Fallot
TR = tricuspid regurgitation
$\mathrm{TV}=$ tricuspid valve

management of TR at the time of PVR have been published so far, with controversial results. ${ }^{5-8}$

In these adult patients with repaired TOF who underwent PVR, we sought to (1) determine factors that predispose to the development of significant preoperative TR and (2) evaluate the efficacy of a concomitant tricuspid valve (TV) repair at the time of PVR.

\section{METHODS \\ Patients}

This was a retrospective study conducted in the Bordeaux University Hospital. Between January 2002 and January 2014, a total of 104 adult (aged $>16$ years) patients (64 male, 40 female) with a repaired TOF or equivalent underwent PVR with insertion of a bioprosthetic valve. Mean and median ages at initial complete correction (corrective surgery) were $20.1 \pm 17.2$ months and 17 months (ranging from 9 to 182) respectively. Mean and median ages at PVR were $26.3 \pm 9.5$ years and 22 years (ranging from 16 to 56), respectively. Thirty-seven patients underwent one palliative procedure before complete correction (7 Waterston shunts, 28 Blalock shunts, and 2 percutaneous PV dilatations). Patients who received a valved conduit during initial correction were excluded from the study. At the time of PVR, 94 patients had severe PR; 10 patients had mixed lesions with moderate stenosis. Demographic data, diagnoses, and procedures are listed in Table 1.

\section{Ethics}

A review of the medical records was undertaken after the institutional ethic committee of Bordeaux University Hospital had approved the study. Patients were informed but a written consent form was not required, according to French law, because the studies were part of the regular management of the patients. No examinations were performed only for the purpose of the study.

\section{Data Collection}

Perioperative data were collected from the hospital records. Demographic and anatomical data, medical history, operative records, and results of transthoracic echocardiography were systematically reviewed. Patient information was collected between July 2014 and December 2014. Duration of the follow-up was defined as the period between surgery and the last clinical visit. All patients had a periodic follow-up with their own cardiologist and additionally were closely followed up in Bordeaux University Hospital with at least one annual visit to obtain clinical details and to undergo a serial echocardiography. No patient was lost to follow-up.

\section{Surgical Indication}

The procedures were performed by 2 experienced surgeons independently. PVR was considered according to the guidelines proposed by
TABLE 1. Characteristics of adults with repaired TOF and who underwent surgical PVR

\begin{tabular}{lc}
\hline \multicolumn{1}{c}{ Variable } & Patients $(\mathbf{n}=\mathbf{1 0 4})$ \\
\hline Sex, $\mathrm{n}(\%)$ & \\
$\quad$ Male & $64(61)$ \\
Female & $40(39)$ \\
Principal diagnosis, $\mathrm{n}$ & \\
TOF & 96 \\
DORV with VSD and PS & 5 \\
TOF with APVS & 1 \\
Critical PS & 2 \\
Age at initial operation, y & $1.67 \pm 1.4$ \\
Trans-annular patch, $\mathrm{n}(\%)$ & $64(62)$ \\
Age at PVR, y & $26.3 \pm 9.5$ \\
Time from initial repair to PVR, $\mathrm{y}$ & $24.8 \pm 9.3$ \\
Aortic cross-clamp time, min, $\mathrm{n}=18$ & $68.1 \pm 23.0$ \\
Cardiopulmonary bypass time, min & $94.3 \pm 48.1$ \\
Moderate or severe TR at PVR, $\mathrm{n}(\%)$ & $41(39.5)$ \\
Associated procedure at PVR, $\mathrm{n}(\%)$ & $44(42)$ \\
TV repair & 16 \\
Pulmonary-branch arterioplasty* & 6 \\
Infundibular aneurism resection & 6 \\
Residual ASD closure & 8 \\
Residual VSD closure & 2 \\
Bentall procedure & 1 \\
AV repair & 1 \\
Pacemaker or cardioverter/defibrillator & 4 \\
\hline Co & \\
\hline
\end{tabular}

Continuous variables are expressed as the mean \pm standard deviation. TOF, Tetralogy of Fallot; DORV, double-outlet right ventricle; VSD, ventricular septal defect; $P S$, pulmonary stenosis; $A P V S$, absent pulmonary valve syndrome; $P V R$, pulmonary valve replacement; $T R$, tricuspid regurgitation; $T V$, tricuspid valve; $A S D$, atrial septal defect; $A V$, aortic valve. *One patient underwent a bilateral pulmonary branch arterioplasty.

$\mathrm{Geva}^{9}$ : when an indexed RV end-diastolic volume at cardiac magnetic imaging (CMR) exceeded $150 \mathrm{~mL} / \mathrm{m}^{2}$, when impaired $\mathrm{RV}$ function was present, or when New York Heart Association (NYHA) functional class III symptoms or worse was present. The decision to perform an associated TV repair for moderate or severe TR was based on the surgeon's preference.

\section{Technique Used for PVR}

Three types of pulmonary valve were inserted into the orthotopic position depending of the surgeon's preference, without a time difference in the use of these pulmonary valves. The implanted valves were the Carpentier-Edwards Perimount bovine pericardial valve (Edwards LifeSciences, Irvine, Calif), the Hancock porcine-valved (DuPont, Wilmington, Del) Dacron conduit (Medtronic, Minneapolis, Minn), and the Medtronic Freestyle valve (Medtronic, Minneapolis, Minn).

\section{Technique Used for TV Repair}

A TV rigid ring annuloplasty was systematically used (MC3, Edwards LifeSciences); the ring was positioned from the anteroseptal commissure to the coronary sinus (Figure 1). When a disrupted commissural chordae at the anteroseptal commissure or septal leaflet distortion secondary to patch closure of ventricular septal defect was found, a concomitant TV suture plasty (commissuroplasty) was added, by approximating together the adjacent edges of the leaflets at this commissure, thus partially obliterating the commissure. 


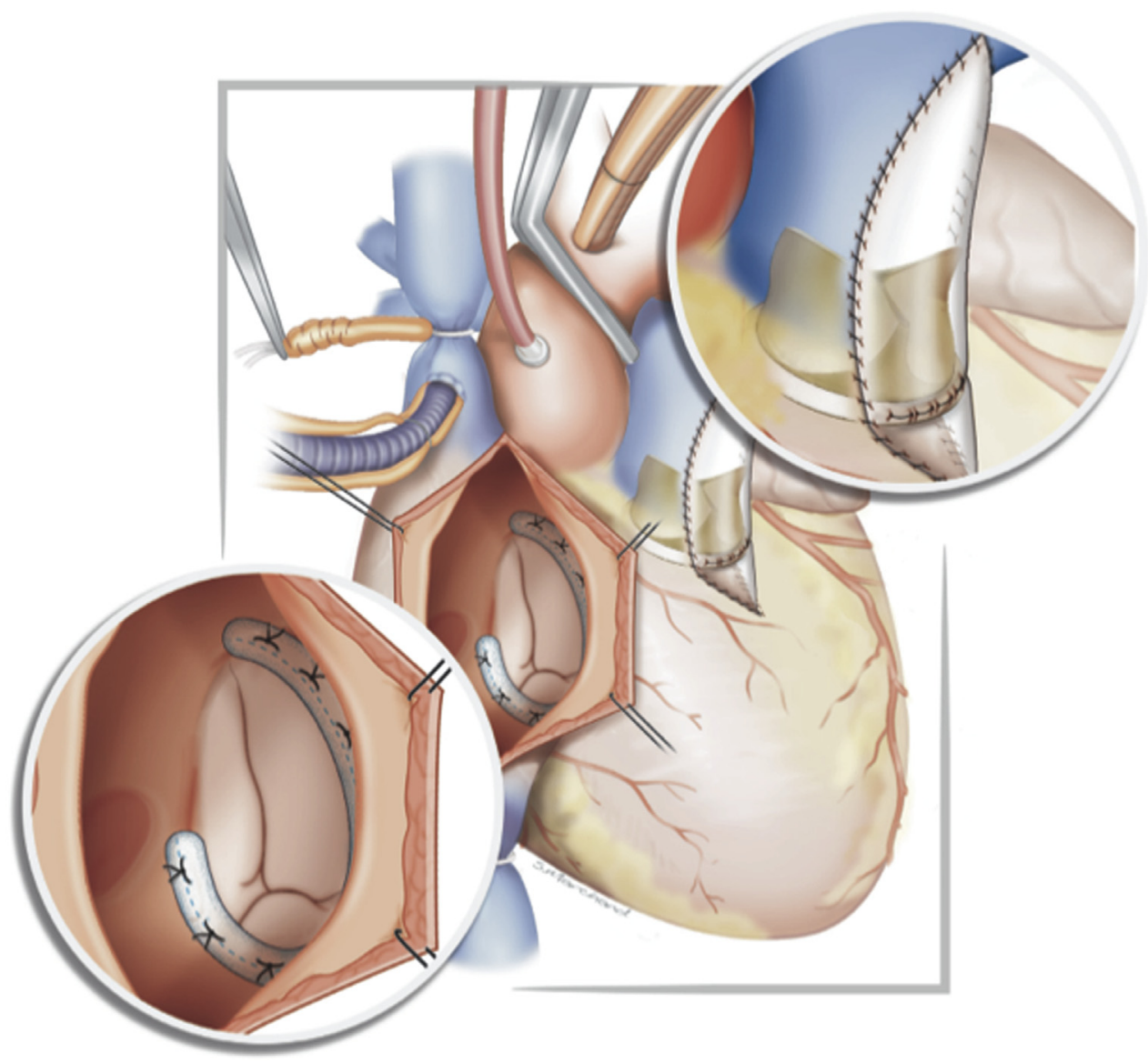

FIGURE 1. Schematic representation of a tricuspid valve repair performed at the time of a pulmonary valve replacement in adults with repaired tetralogy of Fallot. The pulmonary valve replacement is performed by inserting the bioprosthetic valve into the orthotopic position. For the tricuspid valve repair, a rigid ring is positioned from the tricuspid valve anteroseptal commissure to the coronary sinus and is sometimes associated to a commissuroplasty.

\section{Preoperative Features}

To determine whether significant TR occurred early or late after a complete repair, we reviewed echocardiographic studies obtained within 1 year of a complete repair but after initial hospital discharge. The preoperative (before PVR) echocardiographic assessment consisted of measurement of ventricular function, measurement of the maximal instantaneous Doppler gradient across the RVOT, and graduation of PR as none (grade 0 ), mild (grade 1), moderate (grade 2), or severe (grade 3). According to the American Society of Echocardiography guidelines, TR was graded, by use of the width of the vena contracta in an apical 4-chamber view, as none/trivial, mild, moderate, or severe. ${ }^{10}$ Moderate TR and severe TR were considered as significant TR. Significant stenosis of the pulmonary valve was defined as a transvalvular maximal instantaneous Doppler gradient of $>50 \mathrm{~mm} \mathrm{Hg}$ measured along the RVOT. The lateral tricuspid annulus diameter was determined from an apical 4-chamber view. All patients except 4 with a previously implanted pacemaker had a preoperative CMR to measure the pulmonary regurgitant fraction, right and left ventricular end-diastolic and end-systolic volumes, and RV function. All patients with at least one episode of supraventricular (atrial flutter/fibrillation) or ventricular arrhythmia were mapped preoperatively and treated in the catheterization laboratory (radiofrequency ablation). The most common diagnosis was TOF with or without pulmonary valve atresia. All the patients had severe PR or mixed lesions with moderate stenosis (RVOT with a peak gradient $<40 \mathrm{~mm} \mathrm{Hg}$ ). All cases were reoperations, except for one case of TOF with an absent pulmonary valve.

\section{Operative Features}

Initial TOF repair was performed via a transventricular approach. At PVR, all patients underwent a peroperative transesophageal echocardiography. Cardiopulmonary bypass in normothermia was used in all cases, with bicaval cannulation through a redo sternotomy (except in one patient). The operation was conducted with the heart beating for isolated PVR, or PVR associated with pulmonary branch arterioplasty, or resection of a right infundibular aneurysm. Closure of a residual atrial or ventricular septal defect or an aortic-valve replacement was performed with aortic crossclamping and warm-blood cardioplegia. TV repair were performed with or without aortic crossclamping, depending of the surgeon's preference according to the presence of an atrial or a ventricular septal defect. The indication for TV reintervention was severe TR associated with NYHA class III functional status.

\section{Postoperative Features}

Routine postoperative antiplatelet therapy was given for the first 3 postoperative months. To compare with preoperative data, a transthoracic echocardiography was performed 1 year after surgery. Significant postoperative TR was defined as moderate or severe valve insufficiency. CMR was carried out at 1 year postoperatively in 93 patients: 8 patients with a pre- or postoperative implanted pacemaker, 2 deceased patients, and 1 transplant patient did not have postoperative CMR. Transthoracic echocardiography and CMR results were reviewed systematically from the medical records to formulate a database. Routine postoperative cardiac catheterization 


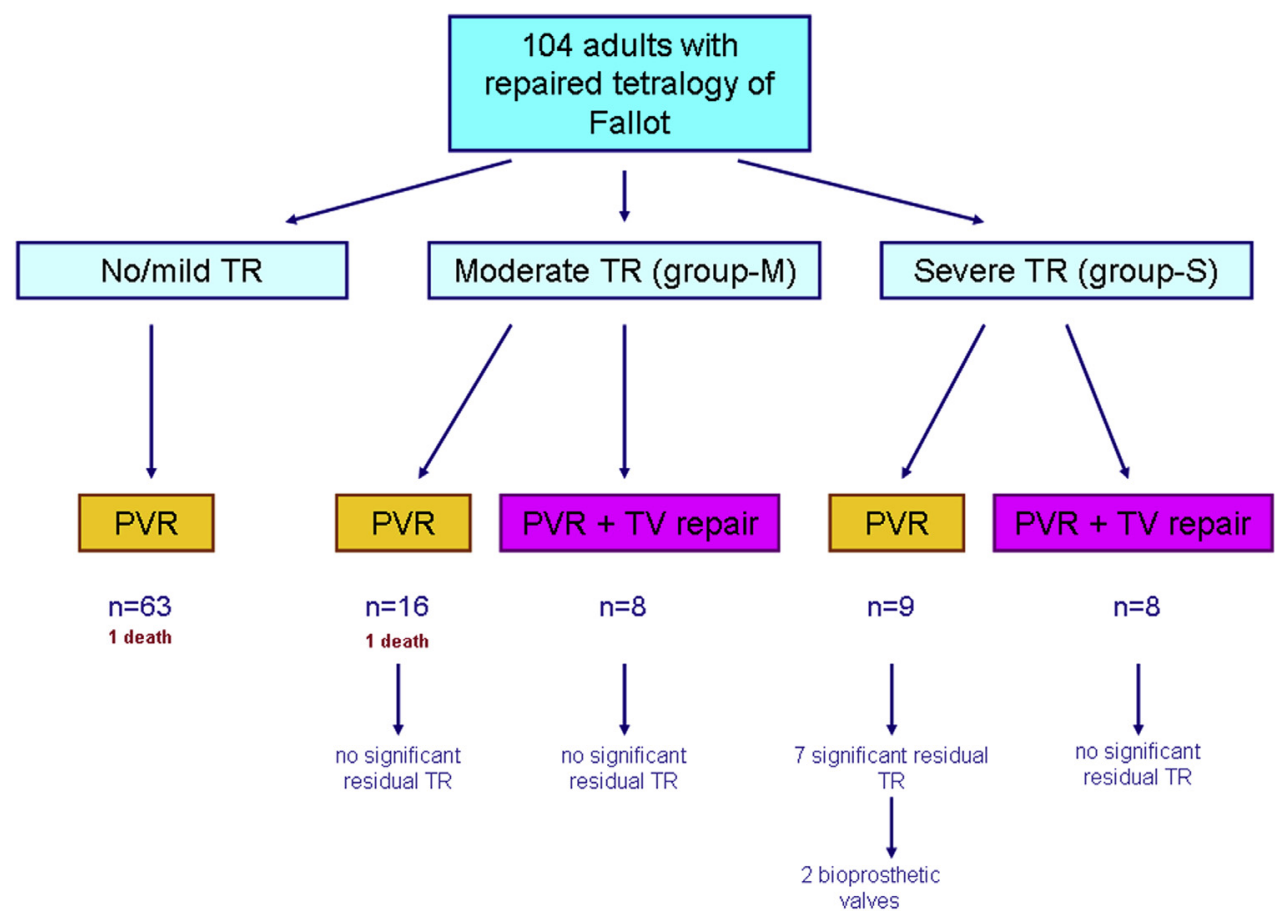

FIGURE 2. Flowchart showing the distribution of the patients according to the functional status of the tricuspid valve. TR, Tricuspid regurgitation; $P V R$, pulmonary valve replacement; $T V$, tricuspid valve.

was not performed. Early postoperative death was defined as any death that occurred within 30 days of surgery or during the same hospital admission. Overall mortality was defined as death occurring from the time of surgery to the most recent follow-up.

\section{Definitions}

Heart failure was classified according to the NYHA functional class. To evaluate the impact of TV repair, we divided the 41 patients with significant (moderate/severe) TR into 2 groups: a moderate group (group M) and a severe group (group S) (Figure 2). For each group, we compared patients who had undergone PVR alone with those who had undergone concomitant TV repair. Pulmonary valve degeneration was defined as a significant pulmonary valve stenosis, a significant regurgitation, or a combination of both. The indication for pulmonary valve reintervention was a gradient of $>50 \mathrm{~mm} \mathrm{Hg}$ or severe regurgitation with RV dysfunction.

\section{Statistical Analysis}

Statistical analysis was performed with the software SPSS 21 (SPSS Inc, Chicago, Ill). Continuous variables are expressed as means \pm standard deviation. To assess their distribution, a Shapiro-Wilk test was used. Then, a Student $t$ test $(\mathrm{n} \geq 20)$ or a nonparametric Wilcoxon $\mathrm{U}$ test $(\mathrm{n}<20)$ was performed to analyze them. A $\chi^{2}$ test $(\mathrm{n} \geq 5)$ or the Fisher exact test $(\mathrm{n}<5)$ was used to analyze categorical variables. To determine preoperative factors that could influence the severity of TR, patients were divided according to the grade of TR and data were compared via a one-way analysis of variance. To analyze the variation of echocardiographic and CMR measurements, a paired $t$ test was used. To analyze the difference of NYHA functional status after PVR, the Fisher exact test was used. The follow-up data on survival, reoperation, and reintervention were analyzed with standard Kaplan-Meier analyses. A log-rank test was used to compare the outcomes of patients who had PVR alone versus patients who had PVR and TV repair. A $P<.005$ was considered statistically significant.

\section{RESULTS \\ Preoperative Factors Associated With TR}

No patient had TR before the first corrective surgery. Mean interval time between initial TOF complete repair and PVR was $24.8 \pm 9.3$ years. No relationship was found between age at TOF repair or age at PVR and the degree of TR (Table 2). The detection of moderate or severe TR in the first year after the initial corrective surgery was associated with the existence of severe TR at PVR $(P<.001)$. Annulus tricuspid valve dilation also was associated with moderate/ severe TR $(P<.001)$. RV volumes, RV function, age at the first corrective surgery, or age at PVR were not significantly associated with moderate/severe TR.

\section{Surgical Characteristics}

Mean cardiopulmonary bypass time was $94.3 \pm 48.1$ minutes (Table 1). Mean aortic crossclamping time (18 patients) was $68.1 \pm 23.0$ minutes. Sixteen patients had a TV repair (of which 10 on the beating heart) with a mean cardiopulmonary bypass time of $111.2 \pm 51.2$ minutes. Mean cardiopulmonary bypass time was $89.3 \pm 47.3$ minutes for patients who did not have concomitant TV repair. Mean prosthetic implanted pulmonary valve diameter was $25 \pm 1.94 \mathrm{~mm}$ (range $21-29 \mathrm{~mm}$ ). At the time of RVOT reconstruction, 32 patients $(31 \%)$ had 1 or 2 additional procedures, for a total of 44 associated procedures $(42 \%)$ at the time of PVR. The most common additional procedure was TV repair in 16 patients (16 
TABLE 2. Preoperative features of adults with repaired TOF at the time of PVR according to the severity of TR

\begin{tabular}{|c|c|c|c|c|}
\hline Variable & $\begin{array}{c}\text { None/mild TR } \\
(\mathrm{n}=63)\end{array}$ & Moderate TR (group M) $(n=24)$ & Severe TR $($ group $S)(n=17)$ & $P$ value \\
\hline Previous systemic-to-pulmonary shunt & $22(35)$ & $8(33)$ & $7(41)$ & .862 \\
\hline Moderate/severe TR at early echo* & 0 & 0 & 8 & $<.001 \dagger$ \\
\hline Age at TOF repair, mo & $18.4 \pm 6$ & $25.5 \pm 7$ & $18.9 \pm 8$ & .221 \\
\hline Age at PVR, y & $26.4 \pm 10$ & $25.3 \pm 9$ & $27.0 \pm 9$ & .830 \\
\hline Interval between TOF repair and PVR, y & $24.8 \pm 9$ & $23.2 \pm 10$ & $25.6 \pm 9$ & .895 \\
\hline $\mathrm{RV}$ end-diastolic volume at $\mathrm{CMR}, \mathrm{mL} / \mathrm{m}^{2}$ & $157.1 \pm 36$ & $163.6 \pm 41$ & $173.8 \pm 26$ & .160 \\
\hline $\mathrm{RV}$ ejection fraction at CMR, \% & $43.1 \pm 10$ & $39.7 \pm 11$ & $40.2 \pm 6$ & .573 \\
\hline Tricuspid annulus diameter, $\mathrm{mm}$ & $29.4 \pm 2.6$ & $33.2 \pm 2.6$ & $36.9 \pm 2.3$ & $<.001 \dagger$ \\
\hline
\end{tabular}

Categorical variables are expressed as effective and percentages in brackets. Continuous variables are expressed as the mean \pm standard deviation. A one-way analysis of variance was used to compare the variables. $T R$, Tricuspid regurgitation; TOF, tetralogy of Fallot; $P V R$, pulmonary valve replacement; $R V$, right ventricle; $C M R$, cardiac magneticresonance imaging. *Moderate or severe TR found at echocardiography in the early postoperative period (first year), following a complete TOF repair. $\dagger P<.005$ was considered statistically significant.

annuloplasty with a flexible ring with 7 additional partial closures of the anteroseptal commissure) (Figure 2). Three patients underwent peroperative epicardial lead and pacemaker implantation for preoperative sinus-node dysfunction.

\section{Postoperative Course}

In-hospital mortality was $1.9 \%$. There were 2 early deaths on postoperative days 17 and 45. Preoperatively, both patients had severe RV dysfunction and moderate left ventricular dysfunction that necessitated postoperative extracorporeal life support but died of multiorgan failure in one case and septic shock in the other. One of these 2 patients had no preoperative TR and the other one had moderate TR. There was no death among the patients who underwent TV repair. There was a $100 \%$ follow-up for the survivors at a mean of $54.6 \pm 34.6$ months (range 6-150). There were no late deaths and survival was $98 \%$ at 1 and 5 years, respectively (Figures 3-5). One patient required orthotopic heart transplantation at 16 months for biventricular heart failure. Two patients with a maximal instantaneous peak gradient of $>50 \mathrm{~mm} \mathrm{Hg}$ at the proximal anastomosis of the Freestyle valve underwent a conduit reintervention (at 13 and 26 months postoperatively). Among the patients with severe TR who underwent PVR alone, 2 suffered symptomatic severe TR during the postoperative course. They both required subsequent TV replacement with a bioprosthetic valve (at 8 and 12 months postoperatively). Event-free survival was $96 \%$ and $91 \%$ at 1 and 5 years, respectively (Figure 5).

\section{Group M Patients}

Among the 24 patients, 8 underwent TV repair and PVR and 16 underwent PVR alone. The preoperative variables were comparable between these two subgroups (Table 3). There was no statistical difference in postoperative variables, and no patient suffered from significant postoperative TR in either subgroup. NYHA score did not seem to be improved after TV repair.

\section{Group S Patients}

Among the 17 patients, 8 underwent TV repair and PVR and 9 underwent PVR alone. The preoperative variables were comparable between these 2 subgroups (Table 4). Whereas there was no significant postoperative TRs in the subgroup who underwent TV repair and PVR, 7 significant postoperative TRs were noted in the group without TV repair $(P=.002)$. Of them, 2 had, for a second time, a TV replacement. For these 2 patients, a TV repair initially was not considered because of multiple previous operations (fourth sternotomies in both). Although all the patients who had TV repair and PVR were classified as NYHA functional class I, only $22.2 \%$ of patients who had PVR alone were classified as class I $(P=.004)$. Thus, in patients with severe preoperative TR, NYHA score seemed to be improved by performing concomitant TV repair.

\section{DISCUSSION \\ Insights from Left-Side Heart Diseases}

Increasing evidence from acquired heart failure studies (secondary to left-sided heart disease, either ischemic or mitral valve disease) suggested that severe functional TR may result in reduced late survival and is associated with the onset of symptoms, atrial fibrillation, and heart failure. ${ }^{11}$ Consequently, TV repair at the time of coronary-artery bypass surgery or mitral-valve surgery may be sometimes beneficial $^{12}$; however, it is still unclear whether the concept of TV repair performed in left-sided heart pathology can be extrapolated to congenital right-sided heart disease.

\section{TR in Repaired TOF}

In adults with repaired TOF, functional TR is common and mainly is due to annulus dilation, which is caused by RV dilation, itself secondary to long-lasting PR. In our series, the incidence of significant TR was $39.5 \%$, which is comparable with previously reported incidences, varying from $19 \%$ to $58 \%{ }^{3-5}$ A recent study highlighted the fact that early reduction (in the first 2-3 postoperative years after PVR) in RV size, progressively deteriorates at 7 to 


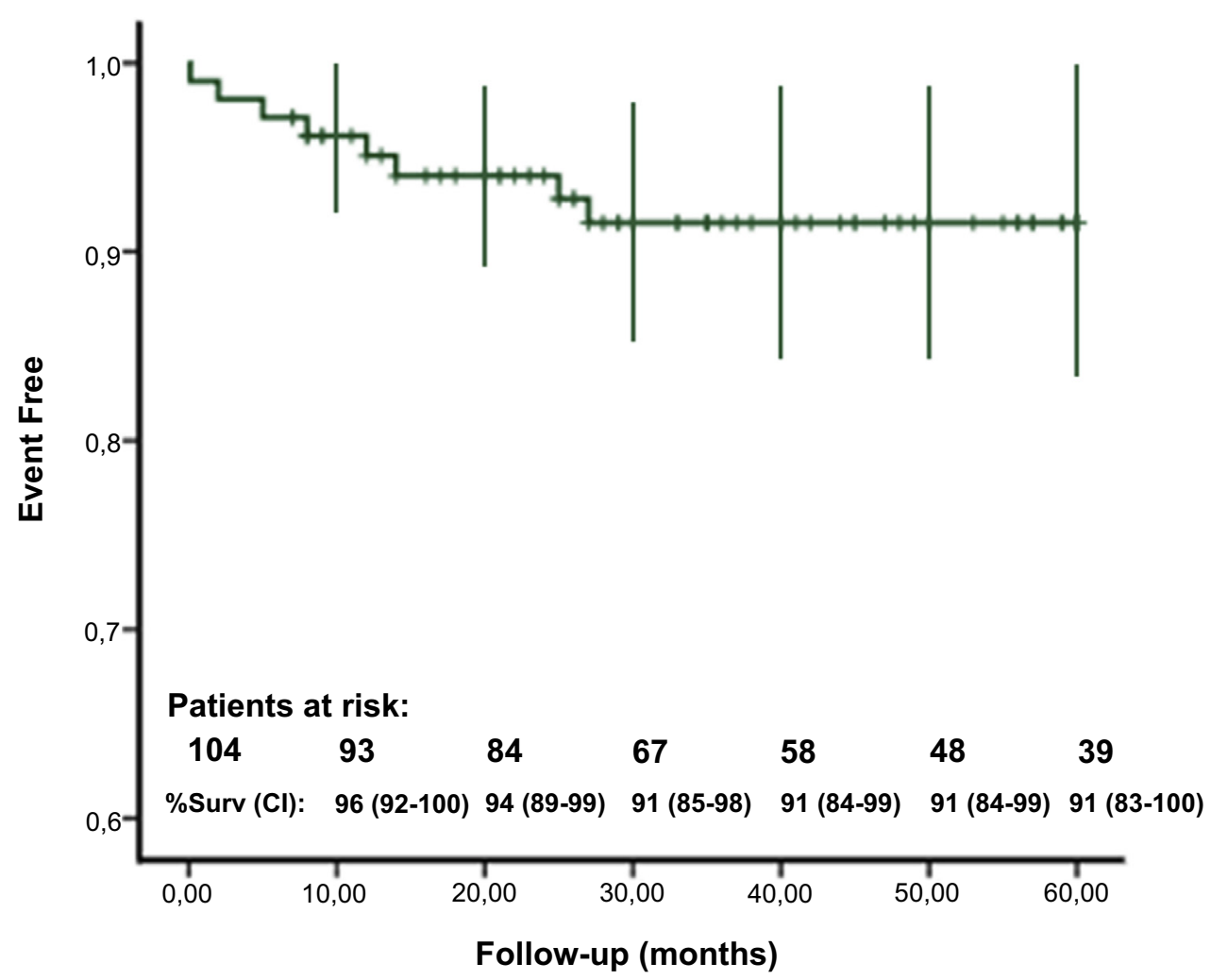

FIGURE 3. Kaplan-Meier curve showing overall survival to events (death, redo pulmonary valve replacement, redo tricuspid valve repair, or heart transplantation) after pulmonary valve replacement in 104 adults with repaired tetralogy of Fallot. Numbers in parentheses represent $95 \%$ confidence intervals. $C I$, Confidence interval.

10 years post-PVR, leading to reverse remodeling and worsened RV function. ${ }^{13}$ This secondary deterioration has been inherent mostly to a subsequent progressive bioprosthetic pulmonary valve dysfunction, but some has been attributed to a TR. ${ }^{13}$

This finding corroborates the fact that TR itself may play a role in the development of RV failure. Thus, Said and colleagues $^{6}$ argued for early PVR before the development or the progression of TR in relation to an annular dilation. As a consequence, these authors systematically perform a TV repair for moderate/severe TR when associated with severe dilation of the tricuspid annulus. ${ }^{6}$ Importantly, the decision for TV repair should be made preoperatively because the conditions of anesthesia generally reduce TR by at least one grade.

In contrast, Cramer and colleagues ${ }^{5}$ reported that TV repair at the time of PVR may not have a clinical benefit, with a similar TR grade at 6 months postoperatively with or without TV repair (in the absence of a clear tricuspid valve abnormality). In this study, however, patients who underwent PVR alone had a slightly lower grade of TR (2.2 vs $2.7, P=.008$ ) and a slightly smaller tricuspid valve annulus diameter, compared with patients who underwent concomitant TV repair.
Similarly, Kogon and colleagues ${ }^{7}$ reported the outcomes of 35 patients who had at least moderate TR at PVR, of whom 16 underwent PVR with concomitant TV repair and 19 underwent PVR alone. The degree of TR and RV dilation decreased significantly at 1 month postoperatively, with or without TV repair, concomitantly with a reduction in RV size and volume. The fact that, for severe TR, however, 8 of the 9 patients underwent an annuloplasty has to be underscored. Nonetheless, leaving a moderate TR at PVR is probably a valuable strategy for secondary (ie, with annulus dilation) moderate TR.

Indeed, in our study, all group $\mathrm{M}$ patients had no significant TR at mid-term, with or without concomitant TV repair. We hypothesize that, PVR being efficient at improving RV volume and function, the change in loading condition explains this improvement. In contrast, for our patients with severe preoperative TR, a TV repair was beneficial. Indeed, at mid-term postoperatively, no patients in group $\mathrm{S}$ who underwent TV repair had significant TR. In a retrospective multicenter study, it was found that a long time after PVR, TR grade remained lower in patients with TV repair $(P<.001) .{ }^{14}$ Moreover, it was stated that patients with severe preoperative TR had a higher risk for adverse events during a 5-year follow-up. At the opposite, Kogon 


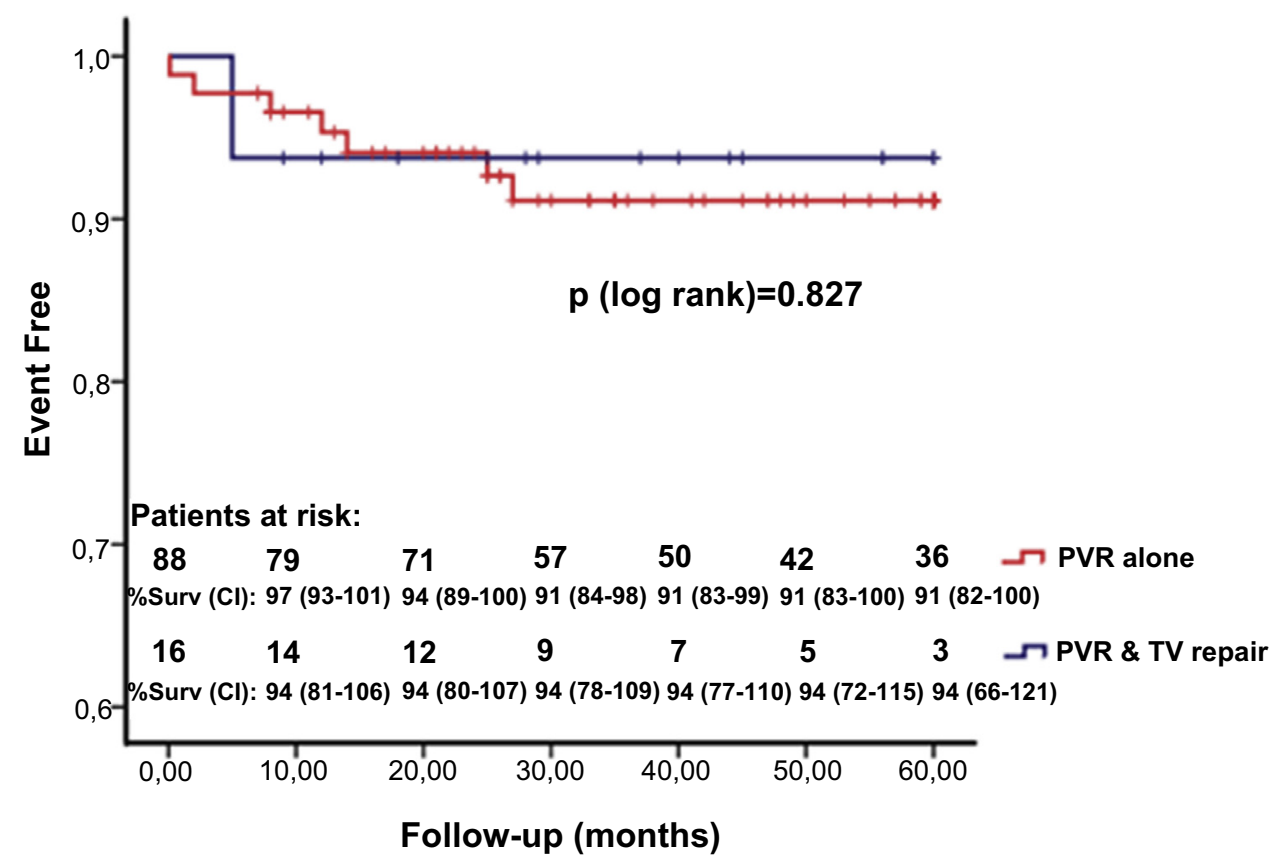

FIGURE 4. Overall survival to events (death, redo PVR, redo TV repair, or heart transplantation) of the entire cohort according to the type of surgery $(\mathrm{n}=104)$. Numbers in parentheses represent $95 \%$ confidence intervals. $C I$, Confidence interval; $P V R$, pulmonary valve replacement; $T V$, tricuspid valve.

and colleagues ${ }^{8}$ suggested that concomitant TV repair may impair long-term tricuspid valve function with a greater degree of TR in patients who undergo PVR with concomitant TV repair. Of note, a semirigid or rigid annuloplasty ring in this study rarely was used $(19 \%)$.

\section{Mechanisms of TR}

When we reviewed the echocardiographic data obtained within the first year after initial complete repair, significant TR was a risk factor for severe TR at the time of PVR. Mahle and colleagues ${ }^{4}$ found that, in some patients,

\section{Follow-up (months)}

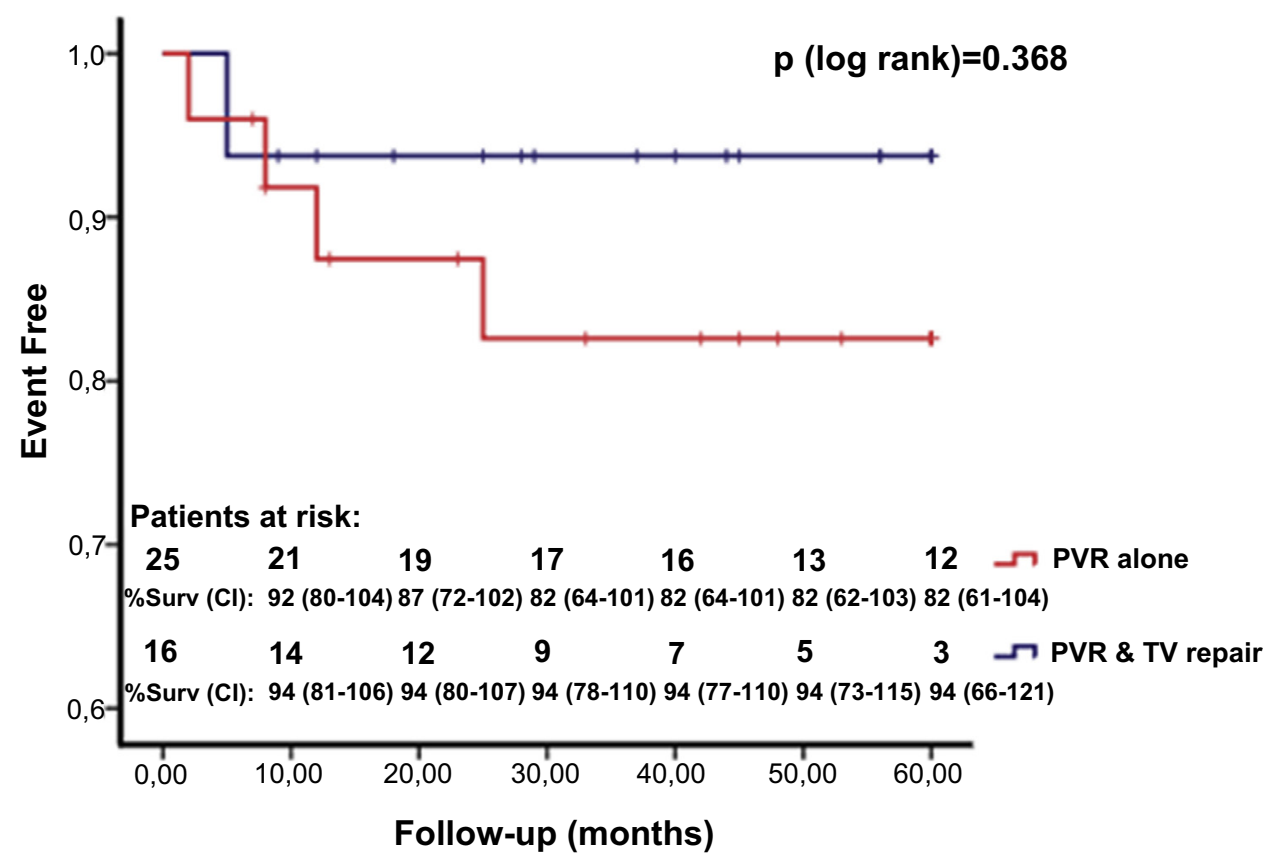

FIGURE 5. Group $M$ and group $S$ survival to events (death, redo PVR, redo TV repair, or heart transplantation) according to the type of surgery ( $\mathrm{n}=41$ ). Numbers in parentheses represent $95 \%$ confidence intervals. $C I$, Confidence interval; $P V R$, pulmonary valve replacement; $T V$, tricuspid valve. 
TABLE 3. Immediate preoperative and 1 year postoperative features of adult patients with moderate TR at the time of PVR (group M) according to the surgical procedure

\begin{tabular}{|c|c|c|c|}
\hline Variable & PVR alone $(n=16)$ & PVR and TV repair $(n=8)$ & $P$ value \\
\hline \multicolumn{4}{|l|}{ Variable: preoperative } \\
\hline Previous palliative surgery, $\mathrm{n}(\%)$ & $5(31)$ & $3(19)$ & 1.00 \\
\hline Age at TOF repair, mo & $28.0 \pm 41$ & $20.4 \pm 8$ & .601 \\
\hline Age at PVR, y & $25.6 \pm 8$ & $24.6 \pm 12$ & .539 \\
\hline Surgical interval, y & $24.8 \pm 8$ & $23.0 \pm 12$ & .283 \\
\hline TR at early echo, $\mathrm{n}^{*}$ & 0 & 0 & NA \\
\hline TV annulus diameter, $\mathrm{mm}$ & $33.3 \pm 3$ & $33 \pm 2.3$ & .951 \\
\hline NYHA class: number of patients $(\%)$ & & & .108 \\
\hline I & $0(0)$ & $0(0)$ & \\
\hline II & $12(75)$ & $3(37.5)$ & \\
\hline III & $4(25)$ & $4(50)$ & \\
\hline IV & $0(0)$ & $1(12.5)$ & \\
\hline RVEF, \% & $41.5 \pm 7$ & $38.1 \pm 8$ & .417 \\
\hline RVESV, mL & $71.3 \pm 17$ & $80.5 \pm 28$ & .645 \\
\hline RVEDV, mL & $160.2 \pm 19$ & $166.4 \pm 33$ & 1.00 \\
\hline \multicolumn{4}{|l|}{ Variable: postoperative } \\
\hline NYHA class: number of patients $(\%)$ & & & .333 \\
\hline I & $16(100)$ & $7(87.5)$ & \\
\hline II & $0(0)$ & $0(0)$ & \\
\hline III & $0(0)$ & $1(12.5)$ & \\
\hline IV & $0(0)$ & $0(0)$ & \\
\hline RVEF, \% & $44.3 \pm 7$ & $43.4 \pm 11$ & .653 \\
\hline RVESV, mL & $49.0 \pm 22$ & $58 \pm 29$ & .706 \\
\hline RVEDV, mL & $86 \pm 32$ & $98 \pm 45$ & .807 \\
\hline Moderate or severe TR, $n$ & 0 & 0 & NA \\
\hline Reoperation for TR & 0 & 0 & NA \\
\hline
\end{tabular}

Continuous variables are expressed as the mean \pm standard deviation. Categorical variables are expressed as number and percentages in brackets. A Wilcoxon $U$ test was used to compare continuous variables and the Fisher exact test to compare categorical variables. $P<.005$ was considered statistically significant. $P V R$, Pulmonary valve replacement; $T V$, tricuspid valve; $T O F$, tetralogy of Fallot; $T R$, tricuspid regurgitation; NA, nonapplicable; NYHA, New York Heart Association; $R V E F$, right-ventricle ejection fraction; $R V E S V$, right-ventricle end-systolic volume; $R V E D V$, right ventricle end-diastolic volume. *Moderate or greater TR found at echocardiography in the early postoperative period (first year), following a complete TOF repair.

significant TR occurred early after a repaired TOF and may have preceded RV dilation. TRs were caused by structural valve abnormalities (tethered septal leaflet and prolapsing or flailing leaflet), and were potentially related to the previous surgical intervention. Those data suggest that 2 mechanisms exist to explain TR: (1) a functional etiology (due to annulus dilation) associated with moderate TR and (2) an iatrogenic etiology caused by chordal disruption or tricuspid septal-leaflet distortion secondary to ventricular septal defect closure at the time of complete TOF repair. The iatrogenic etiology can be worsened by the functional etiology. Consequently, it seems important to avoid TR after a complete TOF repair. In our series, most patients underwent a TOF repair and its subsequent ventricular septal defect closure through the RV. Ventricular septal defect was closed through the right atrium, making it easier to assess the TV and to close the anteroseptal commissure if necessary. Contrary to previous study, we did not find that RV volume was correlated to the development of TR. ${ }^{3}$

\section{Benefit-Risk Balance of TV Repair at the Time of PVR}

The risks and benefits of operating on the tricuspid valve during PVR must be ascertained carefully. Performing a concomitant operation in adults with congenital heart disease is a risk factor for adverse events. ${ }^{15}$ Furthermore, the additional operative time and aortic crossclamp time required to perform TV annuloplasty also should be considered. Antunes and Barlow ${ }^{16}$ stated that concomitant annuloplasty would not add excessive time to a cardiopulmonary bypass and aortic cross clamping. Furthermore, TV surgery with the heart unclamped (either beating or fibrillating) during minimally invasive TV surgery, has already been described. ${ }^{16,17}$ The surgical technique used to repair the TV is relatively easy and straightforward (Video 1). Moreover, congenital abnormalities of the TV have been rarely reported in $\mathrm{TOF}^{4}$ Thus, for isolated functional TR, an annuloplasty is used. In case of iatrogenic lesion, a partial closure of the anteroseptal commissure is performed associated with an annuloplasty 
TABLE 4. Immediate preoperative and 1 year postoperative features of adult patients with severe TR at the time of PVR (group S) according to the surgical procedure

\begin{tabular}{|c|c|c|c|}
\hline Variable & PVR alone $(\mathbf{n}=9)$ & PVR and TV repair $(n=8)$ & $P$ value \\
\hline \multicolumn{4}{|l|}{ Variable: preoperative } \\
\hline Previous palliative surgery, $\%$ & $5(56)$ & $2(22)$ & .335 \\
\hline Age at TOF repair, mo & $20.1 \pm 9$ & $17.3 \pm 2$ & .335 \\
\hline Age at PVR, y & $27.8 \pm 10$ & $26.1 \pm 9$ & .885 \\
\hline Surgical interval, y & $26.2 \pm 9$ & $24.9 \pm 9$ & .809 \\
\hline TR at early echo, $\mathrm{n}^{*}$ & $3(33)$ & $5(55)$ & .347 \\
\hline $\mathrm{TV}$ annulus diameter, $\mathrm{mm}$ & $36.8 \pm 2$ & $37.0 \pm 2$ & .922 \\
\hline NYHA class: number of patients, $\%$ & & & .424 \\
\hline $\mathrm{I}$ & $0(0)$ & $0(0)$ & \\
\hline II & $1(11.1)$ & $3(37.5)$ & \\
\hline III & $7(77.8)$ & $5(62.5)$ & \\
\hline IV & $1(11.1)$ & $0(0)$ & \\
\hline RVEF, \% & $38.3 \pm 5$ & $42.5 \pm 5$ & .347 \\
\hline RVESV, mL & $86.4 \pm 26$ & $75.4 \pm 20$ & .466 \\
\hline RVEDV, $\mathrm{mL}$ & $179 \pm 35$ & $168 \pm 15$ & .379 \\
\hline \multicolumn{4}{|l|}{ Variable: postoperative } \\
\hline NYHA class: number of patients, $\%$ & & & $.004 \dagger$ \\
\hline I & $2(22.2)$ & $8(100)$ & \\
\hline II & $4(44.4)$ & $0(0)$ & \\
\hline III & $2(22.2)$ & $0(0)$ & \\
\hline IV & $1(11.1)$ & $0(0)$ & \\
\hline RVEF, \% & $43.3 \pm 4$ & $46.1 \pm 2$ & .059 \\
\hline RVESV, mL & $53.8 \pm 17$ & $47.7 \pm 14$ & .602 \\
\hline RVEDV, mL & $86.8 \pm 18$ & $80 \pm 9$ & .511 \\
\hline Moderate or severe TR, $n$ & $7(78)$ & 0 & $.002 \dagger$ \\
\hline Reoperation for TR & $2(22)$ & 0 & .47 \\
\hline
\end{tabular}

Continuous variables are expressed as the mean \pm standard deviation. Categorical variables are expressed as number and percentages in brackets. A Wilcoxon $U$ test was used to compare continuous variables and the Fisher exact test to compare categorical variables. $P V R$, Pulmonary valve replacement; $T V$, tricuspid valve; $T O F$, tetralogy of Fallot; $T R$, tricuspid regurgitation; NYHA, New York Heart Association; $R V E F$, right-ventricle ejection fraction; $R V E S V$, right-ventricle end-systolic volume; $R V E D V$, right ventricle end-diastolic volume. *Moderate or greater TR found at echocardiography in the early postoperative period (first year), following a complete TOF repair. $\dagger P<.005$ was considered statistically significant.

ring to stabilize the repair and to reduce the annular diameter, which is often dilated due to long-lasting PR. The application of a rigid (or semirigid) ring has been shown to offer the most durability over time in series that have compared flexible bands with plication annuloplasty techniques (like De-Vega's semicircular annuloplasty technique). ${ }^{18}$ This technique is currently our standard of care in this situation. In our study, the patients in group $\mathrm{S}$ with significant residual TR (none of which have concomitant TV repair) had significantly a worse NYHA status and 2 of these 7 patients underwent in a second time a TV replacement. Considering the fact that TV reoperations are associated strongly with high mortality rates and poor long-term outcomes, evaluating the potential benefits of TV repair is of great importance. ${ }^{16}$

\section{Limits}

This study has several limitations inherent to its retrospective and observational aspect. Indeed, data collection was limited to variables that were available in the medical records. Furthermore, patients were not randomized but chosen based on an individual surgeon's preference and therefore creating an inherent selection bias with regards to performing TV repair or not. Although we have reported the benefits of TV repair during PVR for severe TR over an early- and mid-term follow-up, longer-term studies are needed to determine the longevity of TV repair in this context. Finally, the small size of our cohort (16 patients vs 8 with moderate TR and 9 vs 8 with severe TR) hampers drawing strong conclusions.

\section{CONCLUSIONS}

TR frequently is encountered in adult patients with repaired TOF (or equivalent). Even if its pathophysiology is multifactorial, TR seems to be more severe when present within the first year following the initial corrective surgery. TV repair performed at the time of PVR is safe and feasible. Although TV repair does not seem to be beneficial for patients with moderate preoperative TR, this procedure appears to be of interest only for patients with severe preoperative TR. Thus, in case of severe TR, TV repair, should be systematically considered when PVR is planned. Further studies are needed to confirm the good mid-term outcomes of these patients. 


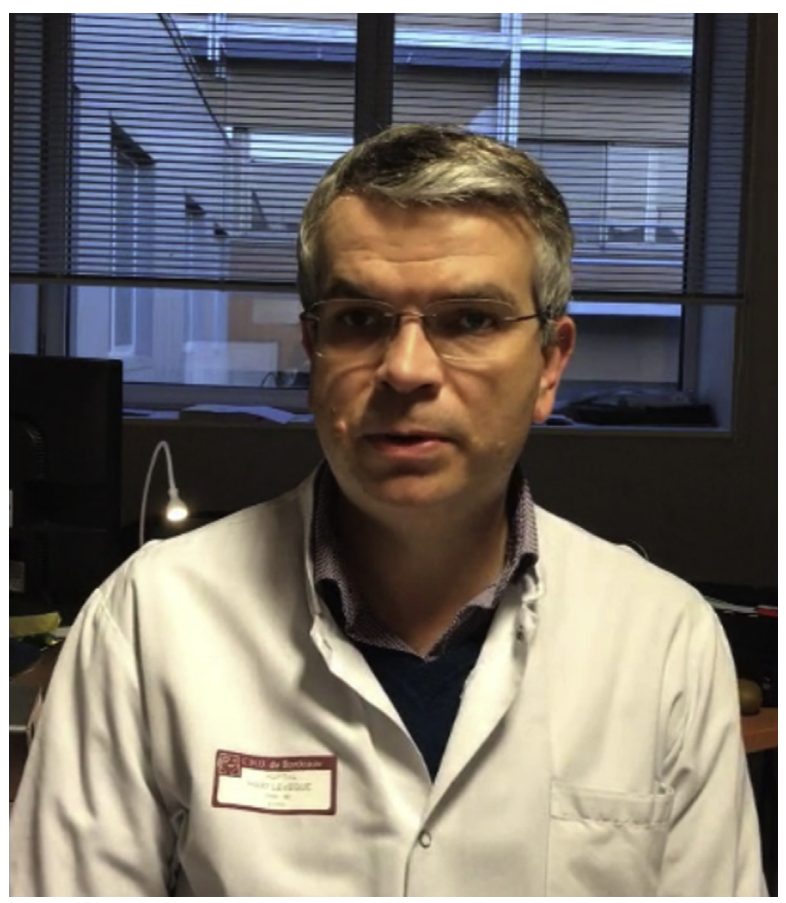

VIDEO 1. Doctor Roubertie presents the interest of performing tricuspid valve repair at the time of pulmonary valve replacement in adult patients with repaired tetralogy of Fallot and severe tricuspid regurgitation. Video available at: http://www.jtcvsonline.org/article/S0022-5223(17)30196-4/ addons.

\section{Conflict of Interest Statement}

Authors have nothing to disclose with regard to commercial support.

\section{References}

1. Cheung MM, Konstantinov IE, Redington AN. Late complications of repair of tetralogy of Fallot and indications for pulmonary valve replacement. Semin Thorac Cardiovasc Surg. 2005;17:155-9.

2. Hawkins JA, Sower CT, Lambert LM, Kouretas PC, Burch PT, Kaza AK, et al. Stentless porcine valves in the right ventricular outflow tract: improved durability? Eur J Cardiothor Surg. 2009;35:600-5.

3. Kobayashi J, Kawashima Y, Matsuda H, Nakano S, Miura T, Tokuan Y, et al. Prevalence and risk factors of tricuspid regurgitation after correction of tetralogy of Fallot. J Thorac Cardiovasc Surg. 1991;102:611-6.
4. Mahle WT, Parks WJ, Fyfe DA, Sallee D. Tricuspid regurgitation in patients with repaired Tetralogy of Fallot and its relation to right ventricular dilatation. Am J Cardiol. 2003;92:643-5.

5. Cramer JW, Ginde S, Hill GD, Cohen SB, Bartz PJ, Tweddell JS, et al. Tricuspid repair at pulmonary valve replacement does not alter outcomes in tetralogy of Fallot. Ann Thorac Surg. 2015;99:899-904.

6. Said SM, Dearani JA, Burkhart HM, Connolly HM, Eidem B, Stensrud PE, et al. Management of tricuspid regurgitation in congenital heart disease: is survival better with valve repair? J Thorac Cardiovasc Surg. 2014;147: 412-7.

7. Kogon B, Patel M, Leong T, McConnell M, Book W. Management of moderate functional tricuspid valve regurgitation at the time of pulmonary valve replacement: is concomitant tricuspid valve repair necessary? Pediatr Cardiol. 2010;31:843-8.

8. Kogon B, Grudziak J, Sahu A, Jokhadar M, McConnell M, Book W, et al Surgery in adults with congenital heart disease: Risk factors for morbidity and mortality. Ann Thorac Surg. 2013;95:1377-82.

9. Geva T. Indications for pulmonary valve replacement in repaired tetralogy of Fallot: the quest continues. Circulation. 2013;128:1855-7.

10. Zoghbi WA, Chambers JB, Dumesnil JG, Foster E, Gottdiener JS, Grayburn PA, et al. Recommendations for evaluation of prosthetic valves with echocardiography and Doppler ultrasound: a report from the American Society of Echocardiography's Guidelines and Standards Committee and the Task Force on Prosthetic Valves, developed in conjunction. J Am Soc Echocardiogr. 2009; 22:975-1014.

11. Nath J, Foster E, Heidenreich PA. Impact of tricuspid regurgitation on long-term survival. J Am Coll Cardiol. 2004;43:405-9.

12. Rogers JH, Bolling SF. The tricuspid valve: current perspective and evolving management of tricuspid regurgitation. Circulation. 2009;119: 2718-25.

13. Hallbergson A, Gauvreau K, Powell AJ, Geva T. Right ventricular remodeling after pulmonary valve replacement: early gains, late losses. Ann Thorac Surg. 2015;99:660-6.

14. Bokma JP, Winter MM, Oosterhof T, Vliegen HW, van Dijk AP, Hazekamp MG, et al. Severe tricuspid regurgitation is predictive for adverse events in tetralogy of Fallot. Heart. 2015;101:794-9.

15. Kogon B, Mori M, Alsoufi B, Kanter K, Oster M. Leaving moderate tricuspic valve regurgitation alone at the time of pulmonary valve replacement: a worthwhile approach. Ann Thorac Surg. 2015;99:2117-22.

16. Antunes MJ, Barlow JB. Management of tricuspid valve regurgitation. Heart 2007;93:271-6.

17. Lee TC, Desai B, Glower DD. Results of 141 consecutive minimally invasive tricuspid valve operations: an 11-year experience. Ann Thorac Surg. 2009;88: 1845-50.

18. Tang GH, David TE, Singh SK, Maganti MD, Armstrong S, Borger MA Tricuspid valve repair with an annuloplasty ring results in improved long-term outcomes. Circulation. 2006;114(suppl):577-81.

Key Words: tetralogy of Fallot, congenital heart disease, pulmonary valve replacement, tricuspid valve repair 